collineation group $G_{216}$ was given by Maschke, Mathematische Annalen, volume 33 , page 317 . Of the five fundamental forms one is a proper sextic curve. Naturally this curve is closely associated with the syzygetic period of cubic curves which the group leaves invariant. Professor Winger discusses the sextic by means of its group property and points out some interesting relations to the system of cubics.

Thomas Buck, Secretary of the Section.

\title{
THE RATIO OF THE ARC TO THE CHORD OF AN ANALYTIC CURVE NEED NOT APPROACH UNITY.
}

BY PROFESSOR EDWARD KASNER.

(Read before the American Mathematical Society, September 9, 1913.)

"IF $P$ is a fixed point on a curve and $Q$ is a point which approaches $P$ along the curve, the limit of the ratio of the arc $P Q$ to the chord $P Q$ is unity." While this statement is frequently made without reservation, it is easy, as in most analogous statements, to construct exceptions in the domain of real functions: by making the curve sufficiently crinkly the limit may become say two, or any assigned number greater than unity.

The object of this note, however, is to point out the necessity for reservation even in the domain of (complex) analytic curves. The limit may then be less than unity. For example, in the imaginary parabola

$$
y=i x+x^{2}
$$

the value of the limit in question, at the origin, is not one, but about .94. The exact value is easily found to be $\frac{2}{3} \nu \overline{2}$.

Of course all such exceptions will be imaginary. Thus for a real non-circular ellipse the limit is obviously unity at each of the $\infty^{1}$ real points; but of the $\infty^{2}$ imaginary points of the ellipse, there are four points at which the limit takes the value $.94+$. These are the points at which the tangent is a minimal (or isotropic) line. Thus if we explore all the points of the ellipse, 
in the complex domain, the limit takes the usual value at all points except four points where the value is .94. The limit is therefore a discontinuous function of the point of the curve.

\section{Regular Elements or Curves in the Plane.}

The general theory is very simple. Consider first any analytic curve in the plane in the neighborhood of a point $P$ at which it is regular. The equation of the curve referred to rectangular axes through $P$ can be taken in the form

$$
y=c_{1} x+c_{2} x^{2}+c_{3} x^{3}+\cdots,
$$

where the coefficients $c$ are complex numbers.

The chord $\gamma$ from the point $P(0,0)$ to the neighboring point $Q(x, y)$ is

$$
\begin{aligned}
\gamma & =\sqrt{x^{2}+y^{2}} \\
& =x \sqrt{1+c_{1}^{2}+2 c_{1} c_{2} x+\left(2 c_{1} c_{3}+c_{2}^{2}\right) x^{2}+\cdots} .
\end{aligned}
$$

The length of the arc is

$$
\begin{aligned}
\alpha & =\int_{0}^{x} \sqrt{1+{y^{\prime}}^{2}} d x \\
& =\int_{0}^{x} \sqrt{1+c_{1}{ }^{2}+4 c_{1} c_{2} x+\left(6 c_{1} c_{3}+4\right.} \frac{\left.c_{2}{ }^{2}\right) x^{2}+\cdots}{d x} .
\end{aligned}
$$

If the term $1+c_{1}^{2}$ does not vanish, that is, if the slope $c_{1}$ does not equal $\pm i$, then $\gamma$ and $\alpha$ can be developed into integral power series starting with the first power of $x$, the coefficient of $x$ in both cases being $\sqrt{1+c_{1}^{2}}$. Letting $x \doteq 0$, we thus have the usual result

$$
L \equiv \lim \alpha / \gamma=1 .
$$

If, on the other hand, $1+c_{1}{ }^{2}$ does vanish, the developments of $\gamma$ and $\alpha$ are series in fractional powers of $x$. Assuming that $c_{2}$ does not vanish, the leading term in $\gamma$ is then

and that in $\alpha$ is

$$
\sqrt{2 c_{1} c_{2}} x^{\frac{3}{2}}
$$

$$
\int_{0}^{x} \sqrt{4 c_{1} c_{2} x} d x=\frac{2}{3} \sqrt{4 c_{1} c_{2}} x^{\frac{3}{3}},
$$


where $c_{1}$, of course, is $\pm i$. Then our limit becomes

$$
L=\frac{2}{3} \sqrt{2}=.94+\text {. }
$$

If however $c_{2}=0$, in addition of course to $1+c_{1}{ }^{2}=0$, while $c_{3} \neq 0$, the leading terms in (2) and (3) are

and

$$
\sqrt{2 c_{1} c_{3}} x^{\frac{5}{2}}
$$

$$
\int \sqrt{6 c_{1} c_{3}} x^{2} d x=\frac{1}{2} \sqrt{6 c_{1} c_{3}} x^{\frac{5}{2}}
$$

so that

$$
L=\frac{1}{2} \sqrt{3}=.86+.
$$

In the most general exceptional curve, we have say $c_{1}= \pm i$, $c_{2}=0, c_{3}=0, \cdots, c_{k-1}=0, c_{k} \neq 0$, so that the equation of the curve is of the form

$$
y= \pm i x+c_{k} x^{k}+c_{k+1} x^{k+1}+\cdots \quad\left(c_{k} \neq 0\right) .
$$

The curve then has contact of order $k-1$ with its minimal tangent. We find then

$$
\begin{gathered}
\gamma=\sqrt{2 c_{1} c_{k}} x^{\frac{k+1}{2}}+\cdots, \\
\alpha=\frac{2}{k+1} \sqrt{2 k c_{1} c_{k}} x^{\frac{k+1}{2}}+\cdots,
\end{gathered}
$$

so that

$$
L=\lim \frac{\operatorname{arc}}{\text { chord }}=\frac{2 \sqrt{k}}{k+1} .
$$

Theorem I. The limit of the ratio of the arc to the chord at a regular point of an analytic curve is unity provided the tangent at that point is not a minimal line. In the latter event (that is, when the slope is $\pm i$ ) the limit is given by (7), where $k-1$ denotes the order of contact of the curve with the minimal tangent.*

In the trivial case where the given curve is a minimal line (instead of merely touching a minimal line), the limit does not exist.

Omitting this trivial case, we see that at every point, real

* That is, $k$ is the number of consecutive points common to the curve and the tangent. In this form we observe that (7) remains valid even when $k=1$, for then $L=1$. 
or imaginary, the limit exists and is always real. If it is not one, it is at most .94 + ; if it is less than this, it is at most $.86+\cdots$. Thus the limit value $L$ is always a number selected from the denumerable set

$$
\frac{2 \sqrt{k}}{k+1} \quad(k=1,2,3, \cdots)
$$

the first few terms being, to two decimal places,

$$
1.00, .94, .86, .80, .74, \quad \cdots .
$$

Most of these numbers are irrational, but some are rational. Thus in the case of third order contact with the minimal line, that is, $k=4$, we have $L=4 / 5$.

Notice that in all the exceptional cases $L$ is less than unity, that is, the arc becomes less, in absolute value, than the chord. This is as we should expect. For the chord ultimately diverges more from the minimal tangent than does the arc, and along a minimal line distances are zero.

The sequence (8) has zero for its limit. That is, if $k$ is made larger and larger, so that the curve has higher and higher contact with the minimal tangent, the value of $L$ approaches. zero. But, as observed before, if we actually let the curve reduce to a minimal line, the limit $L$ ceases to exist. Therefore $L$ cannot be made equal to zero; this number is not contained in the sequence (8); but $L$ can be made smaller than any assigned number.

\section{Irregular Elements.*}

Consider next irregular analytic arcs, that is, curves which, in a neighborhood of the given point $P$, taken as origin of rectangular axes, cannot be represented (for any choice of axes) by setting $y$ equal to a series in integral powers of $x$, but can be represented by a series with fractional exponents, say a series arranged in powers of the $p$ th root of $x$. If the slope of the curve at $P$ is not $\pm i$, the usual value $L=1$ is obtained. Otherwise we have the following

Theorem II. If the analytic curve is irregular at the point $P$, and if the tangent line is minimal, the equation of the curve,

* We are dealing always with an analytic element, that is, an analytic curve at a given point. We apply the adjective irregular either to the point or the curve or (preferably) the element. Cf. the usage of Study, $\$ 6$. 
referred to $P$ as origin, being of the form

$$
y= \pm i x+c_{p+k-1} x^{\frac{p+k-1}{p}}+c_{p+k} x^{\frac{p+k}{p}}+\cdots,
$$

the limit of the ratio of the arc to the chord is

$$
L=\frac{2 p}{2 p+k-1} \sqrt{\frac{p+k-1}{p}} .
$$

For the regular curves we have, of course, $p=1$, so that this general formula (10) reduces to (7). The range of possible values of $L$ for the irregular arcs is greater, since the result (10) depends on the two integers $p$ and $k$, characterizing the type of irregular (singular) point and the contact with the minimal tangent. The set of numbers (10) is dense everywhere between 1 and 0 , while set (7) is dense only at 0 . The value of the limit $L$ may, in particular, now lie between 1 and .94. Thus for an ordinary cusp with minimal tangent, that is, $p=2, k=2$, we have

$$
L=\frac{4}{5} \sqrt{\frac{3}{2}}=.96+.
$$

If $p$ is fixed and $k$ is made large, $L$ becomes nearly 0 ; while if $k$ is fixed and $p$ made large, $L$ becomes nearly 1 .

We observe that $L^{2}$ in (10) is always rational and not greater than one. Not every proper fraction, however, can be a value of $L^{2}$. The necessary and sufficient condition is that $1-L^{2}$ shall be the square of a proper fraction.

\section{A Real Representation of the Results.}

Is it possible to bring the exceptional cases we have found within the realm of intuition instead of mere calculation? Can we picture, for example, the limiting ratio .94? One plan would be to use Study's real representation of analytic curves by means of $\infty^{2}$ point pairs. The interpretation of distance and arc is then of course real but quite complicated.* We therefore use an indirect plan which is more convenient for our particular purpose.

We pass from the original $(x, y)$ plane to a $(u, v)$ plane by the imaginary affine correspondence

$$
u=x+i y, \quad v=x-i y .
$$

* See Study, Vorlesung über ausgewählte Gegenstände der Geometrie, Erstes Heft (1911), §2. 
The element of arc in the $(x, y)$ plane,

$$
d s=\sqrt{d x^{2}+d y^{2}},
$$

then becomes

$$
d \sigma=\sqrt{d u d v}
$$

Thus the distance between two points $P$ and $Q$ in the first plane becomes the "pseudo-distance" $P^{\prime} Q^{\prime}$, defined not as the length of the segment $P^{\prime} Q^{\prime}$, but as the side of a square having the same area as the rectangle constructed with $P^{\prime} Q^{\prime}$ as diagonal, the sides being parallel to the $u, v$ axes.

The pseudo-length of an arc running from $P^{\prime}$ to $Q^{\prime}$ is of course obtained by inserting many intermediate points and constructing on the sides of the inscribed broken line little rectangles having those sides as diagonals, then adding, not the areas of these rectangles (that would give merely zero in the limit) but the square roots of the areas. The pseudolength of the chord $P^{\prime} Q^{\prime}$ is obtained from the single big rectangle constructed for the diagonal $P^{\prime} Q^{\prime}$.

With these definitions, we readily find that the ratio of the pseudo-arc to the pseudo-chord approaches unity when $Q^{\prime}$ approaches $P^{\prime}$, except when the tangent line at $P^{\prime}$ is horizontal or vertical. In fact the minimal lines in the $(x, y)$ plane are converted by our representation (11) into the lines parallel to the $u, v$ axes. If we take a real curve in the $(u, v)$ plane touching the horizontal line at $P^{\prime}$, the pseudo-arc $P^{\prime} Q^{\prime}$ will ultimately become smaller than the pseudo-chord $P^{\prime} Q^{\prime}$. If the contact is of the first order, the limiting value of the ratio is $.94+$, a result that could be verified by an accurate draughtsman. The other values of $L$, corresponding to higher contact and irregular points according to formulas (7) and (10), would be obtained with increasing experimental difficulties.

\section{Space Curves.}

So far we have been considering only plane curves. For analytic curves in space (of three or more dimensions) the results are quite different. The limit of the arc to the chord is of course unity for all real curves; and also for all complex curves provided the tangent line at the given point is not minimal. For the exceptional curves, however, the range of possibilities is much greater than in the plane. In the plane curve, the limit is always real. For space curves it may be 
imaginary. In the plane, when the limit is not unity, the arc ultimately becomes less in absolute value than the chord. In space it may become either less or greater. While the set of possible values for $L$ in the plane is denumerable, it constitutes, in space, a continuum. This is true even if we confine ourselves to regular analytic curves.

To justify these statements, we need not give a complete classification, but may confine ourselves to the special category of curves

$y=a_{1} x+a_{2} x^{2}+a_{3} x^{3}+\cdots, \quad z=b_{1} x+b_{2} x^{2}+b_{3} x^{3}+\cdots$

defined by the relations

$$
\begin{gathered}
1+a_{1}^{2}+b_{1}^{2}=0, \quad a_{1} a_{2}+b_{1} b_{2}=0, \\
\left(a_{2}^{2}+b_{2}^{2}\right)\left(a_{1} a_{3}+b_{1} b_{3}\right) \neq 0 .
\end{gathered}
$$

By a simple calculation of the leading terms in the developments for arc and chord we find for such a curve

$$
L=\sqrt{\frac{3 \lambda+2}{4 \lambda+2}},
$$

where

$$
\lambda=\frac{a_{1} a_{3}+b_{1} b_{3}}{a_{2}{ }^{2}+b_{2}{ }^{2}} .
$$

Here $L$ does not depend merely upon certain integers (arithmetic invariants, order of contact), as was the case in the plane, but upon the actual coefficients in the equations of the curve, that is, upon differential invariants. Since $\lambda$ may take any complex value, the same is true for $L$.

Theorem III. Regular analytic curves in space can be constructed for which, at a given point, $L$ shall have any assigned real or imaginary value.

\section{Relation to Conformal Geometry.}

It is worth while pointing out that the quantity $L$ defined for any point of an analytic curve is important not only in metric geometry, based on the group of displacements, but in the more general conformal geometry, based on the group of all conformal transformations, a group which is infinite in the plane and finite in space. This follows from the fact that the value of $L$ depends on the relation of the given curve to 
minimal lines and curves. In fact the conformal group may be defined as consisting of all point transformations for which $L$ is invariant. In particular it is found that two regular elements in the plane are conformally equivalent when and only when they agree with respect to $L$.

This is not true of irregular arcs. The complete discussion of the conformal invariants of irregular analytic curves, or elements, will be given in another paper. The main result is that every such curve has differential invariants (certainly more than one, and probably an infinite number) except in the type represented by an ordinary cusp with non-minimal tangent. This type can be reduced formally to the normal form $y=x^{\frac{3}{2}}$, and therefore has no invariant. Furthermore, any such analytic cusp can be transformed into any other by a unique conformal transformation.

The power series defining an analytic element may be real or imaginary, integral or fractional. If, furthermore, we allow the series to be divergent as well as convergent, we have also a classification of elements as divergent or convergent. The concept of divergent differential element of infinite order thus introduced promises to lead to greater simplicity and generality in the differential geometry of curves and surfaces. The resulting "geometry of divergent power series" will be treated elsewhere.

Columbia University, NeW YoRK.

\section{A MERSENNE PRIME.}

By Lucas's theorem (American Journal of Mathematics, volume 1, page 305) a Mersenne number $n=2^{4 q+3}-1$ with $4 q+3$ a prime and $8 q+7$ composite is a prime number if the first term of the series $3,7,47,2207, \ldots$ divisible by $n$ lies between the $(2 q+1)$ th and the $(4 q+2)$ th. My computations show that $2^{107}-1$ divides the 106 th term of the series and is therefore a prime number.

Denver, Colo.

R. E. Powers. 